\title{
Wem gehört die Planung? Recht auf Stadt jenseits des Kolumbus-Syndroms \\ Kommentar zu Lucius Burckhardts „Wer plant die Planung?“
}

Kommentare von:

Wolf Reuter, Beate Binder, Jens S. Dangschat, Monika Grubbauer, Michael Guggenheim, Stadt von Unten, Iris Dzudzek

Iris Dzudzek

\section{Einleitung}

Mit spitzer Feder beschreibt Lucius Burckhardt in seinem Aufsatz „Wer plant die Planung?" die konfligierenden Rationalitäten der am Planungsprozess beteiligten Akteure. Dabei zeigt er auf, wie „das Kräfteparallelogramm zwischen der regierenden Beamtenschaft, der Bauspekulation, der Bürgerschaft und den durch die beschlossenen Maßnahmen betroffenen Leute“ (S. 107) die „Übelstände der Stadt“ häufig verschlimmbessert. Es fehle ein „strategisches Vorgehen“, das „dem Systemcharakter der Stadt angemessen" (S. 113) wäre.

Burckhardts Beobachtungen der Machtverquickungen von Planer*innen sind scharfsinnig und - wie sich auch später noch zeigen wird - in vielen Punkten visionär. Er erkannte bereits damals, dass die Gestaltungsmöglichkeiten von Beteiligten in den von der Planungstheorie entwickelten Partizipationsverfahren zur reinen Organisation von Zustimmung verkommen (vgl. S. 112). Allerdings reproduziert er die Trennung aller am Planungsprozess Beteiligten in Planer*innen und Beplante, das bedeutet, in diejenigen, die die Stadt aktiv gestalten dürfen und diejenigen, die ihre Veränderungen passiv zu erdulden haben. Die Haltung, die dieser Trennung zugrunde liegt, werde ich als ,Kolumbus-Syndrom“ bezeichnen. Betroffene wie auch die Stadt lassen sich aber nur bedingt am Reißbrett formen. Demgegenüber und in Fortführung von Ansätzen, wie sie Burckhardt später anhand performativer Installationen und der Promenadologie entwickelt hat (Burckhardt 2013; 2006, siehe auch Gribat 2017), vertritt der vorliegende Kommentar die These, Planung sollte bei den vorhandenen Ressourcen und aktuellen Nutzungen in der Stadt ansetzen. Dies gilt ganz besonders in Zeiten schrumpfender Freiräume in der unternehmerischen Stadt, die mit massiven sozialen und ökologischen Problemen zu kämpfen hat. Hierzu gilt es, Partizipation aus ihrer postpolitischen Einhegung in Planungsprozessen herauszuholen. Dabei ist nicht nur eine Neuerfindung und Repolitisierung der Partizipationsprozesse notwendig, sondern auch ein Umdenken in der Planung. Diese sollte weniger an neuen Entwürfen als an der bereits in 
der Stadt gelebten Praxis und ihren häufig prekären Raumproduktionen ansetzen. Planung kann hier viel vom postkolonialen Urbanismus sowie von performativen und ästhetischen Ansätzen lernen. Die Rolle der Planung wäre dann weniger die Bereitstellung und Umsetzung von Entwürfen, sondern vielmehr eine Weiterentwicklung und Moderation bereits gelebter Raumproduktionen, die ein Recht auf Stadt für alle realisiert.

\section{Veränderungen}

Das städtebauliche Leitbild, das Burckhardt vor Augen gehabt haben dürfte, als er 1974 „Wer plant die Planung?" schrieb, war das der funktional getrennten, autogerechten Stadt mit hohem Flächenverbrauch mit den bekannten ökologischen Folgen. Seither haben sich die Gesellschaft und mit ihr auch die Paradigmen der Planung verändert. Das Leitbild der autogerechten und funktionalen Stadt ist der funktional durchmischten Stadt der kurzen Wege gewichen. Das gesellschaftliche Wunschbild eines Häuschens im suburbanen Grün ist jenem der Altbauwohnung inmitten einer kreativen Arbeits- und Freizeitszene gewichen. Die integrierte Entwicklungsplanung der 1960er und 1970er Jahre und die kleinteilige Stadterneuerung mit Bürgerbeteiligung der 1970er wurden in den 1980ern vom perspektivischen Inkrementalismus abgelöst (Sieverts/Ganser 1993). Die Renaissance strategischer Planung der 1990er Jahre setzte dann stärker als ihre Vorgängerversion der 1970er auf eine Steuerung durch marktwirtschaftliche Modelle und die Übertragung von betriebswirtschaftlichen Planungsmethoden auf den öffentlichen Bereich (new public management). Damit reagierte die Planung auf die neuen räumlichen Bedürfnisse postfordistischer Arbeitswelten und Konsummuster des interkommunalen Wettbewerbs sowie der Urbanisierung des Kapitals (Harvey 2009) und wurde zur Kooperationspartnerin neoliberaler Stadtentwicklung. Eine neue Kaste von policy entrepreneurs bereist seither die Welt. Sie verkaufen Politikmodelle, die zu global zirkulierenden blue prints werden und Städte immer ähnlicher aussehen lassen (Peck 2011, Prince 2014). Richard Floridas „Kreative Stadt“ (Florida 2002) dürfte das prominenteste Beispiel dieser Art sein (Dzudzek 2016).

Beeindruckend ist, mit welch analytischer Schärfe Burckhardt Entwicklungen problematisiert, die 1974 noch in ihren Anfängen steckten und bis heute an Brisanz gewonnen haben. Zu nennen ist hier die „Wiederaufwertung des Zentrums“ (S. 107) und die Umwandlung „,städtischer Liegenschaften [in] Handelsware“ (S. 106). Selbige prägen bis heute Prozesse der Gentrifizierung und Verdrängung in der Stadt und haben im Zuge von Neoliberalisierung und der Verschärfung des interkommunalen Wettbewerbs an Dringlichkeit gewonnen (Schipper 2013). Ein weiterer interessanter Punkt findet sich in Burckhardts Skepsis gegenüber den damals neuen Formen der Bürgerpartizipation. Diese kritisiert er als Fortführung der Methoden, mit denen sich die Verwaltung seit dem Ancien Regime Zustimmung verschaffe (vgl. S. 111). „Die Förderung“, die die Planung laut Burckhardt der Bürgerbeteiligung ,angedeihen lässt, ist identisch mit der Zerstörung des emanzipatorischen Werts dieser Veranstaltung“ (S. 112). In der Tat haben sich die Partizipationsmodelle seit den 1970er Jahren rasant weiterentwickelt und leider hat sich Burckhardts Sorge bestätigt: Bürgerbeteiligung ist zum 
„Herrschaftsinstrument“(Wagner 2013) geworden, das den postpolitischen Konsens unternehmerischer Stadtpolitik prozessiert (Rosol/Dzudzek 2014, Gomes de Matos 2013, Dzudzek 2013).

\section{Das Kolumbus-Syndrom}

Persistent ist auch die von Burckhardt beschriebe „Intuition“ (S. 107) von Architekt*innen und Planer*innen, die zwar „das vorgelegte Problem auf das ,Wesentliche““ (ebd.) reduziert, die allerdings auch vom „planerischen Kontext“ (ebd.) abstrahiert. Diese planerische Praxis, die Burckhardt so treffend in seinem Aufsatz beschreibt, beruht auf Entwürfen. Sie werden vorzugsweise auf weißen Leinwänden angefertigt. Nicht selten sprechen die Selbstdarstellungen und Projekte von Architekt*innen hier Bände. Ein Haus oder ein städtebauliches Ensemble steht allein vor einem weißen oder monochromen Hintergrund. Sofern städtischer Kontext vorkommt, wird er im rendering gern ausgeblasst oder nur schemenhaft angedeutet. Die technokratische Vision der Formung eines Gemeinwesens durch den Entwurf neuer Lebens- und Arbeitswelten, die den Kern von Planung bildet, ist hier versinnbildlicht. Und selbst die Planung, die sich stärker dem städtebaulichen Kontext widmet, beruht häufig auf Visionen und setzt selten konkret am jeweiligen Ort und der dort stattfindenden Praxis an.

In dieser Vorstellung von Planung versteckt sich eine Machtasymmetrie zwischen den Planner*innen, die wissen, was für die passivierten Betroffenen das ,Wesentliche“ ist. Dies stattet erstere mit der Autorität aus, die Stadt mit ihren Visionen ,beplanen` zu dürften. Paradigmatisch dafür ist, dass auch in Burckhardts Text die „Beplanten“ erst auf Seite 11 von 13 auftauchen. Diese Machtasymmetrie, die in der Planungspraxis bis heute fortlebt, möchte ich als ,Kolumbus-Syndrom‘ bezeichnen. Es war der schwarze Filmemacher Spike Lee, der diesen Begriff bei einem Auftritt im New Pratt Institute for Black History und in Reaktion auf Gentrifizierungs- und Verdrängungsprozesse in Brooklyn prägte:

„Then comes the motherfuckin' Christopher Columbus Syndrome. You can't discover this! We been here. You just can't come and bogart. There were brothers playing motherfuckin' African drums in Mount Morris Park for 40 years and now they can't do it anymore because the new inhabitants said the drums are loud. My father's a great jazz musician. He bought a house in nineteen-motherfuckin'-sixty-eight, and the motherfuckin' people moved in last year and called the cops on my father. He's not - he doesn't even play electric bass! It's acoustic! We bought the motherfuckin' house in nineteen-sixty-motherfuckin'-eight and now you call the cops? In 2013? Get the fuck outta here!

Nah. You can't do that. You can't just come in the neighborhood and start bogarting and say, like you're motherfuckin' Columbus and kill off the Native Americans. Or what they do in Brazil, what they did to the indigenous people. You have to come with respect. There's a code. There's people." (Lee in Coscarelli 2014)

Das Kolumbus-Syndrom lässt Menschen glauben, sie hätten einen Ort gerade erst entdeckt. Dabei übersehen siejene, die dort bereits leben oder arbeiten. Sie 
eignen sich diese Orte an, weil sie glauben, sie würden von niemandem beansprucht. In diesem Sinne ist Planung häufig übergriffig oder gewaltförmig. Sichtbar wird dies besonders in der Umsetzung. Zu nennen sind hier nicht nur die Prozesse der Verdrängung von Bevölkerung im Zuge von Aufwertung und state-led gentrification. Meines Erachtens kann der Begriff auf weitere, sehr unterschiedliche Praktiken übergriffiger Planung ausgedehnt werden. Dazu gehört die Zerstörung selbstgebauter Unterkünfte von Roma in Frankfurt ebenso wie das Kunstprojekt xqm. Es erschloss „spezifische, selten bewußt genutzte Bereiche im innerstädtischen öffentlichen Raum der Stadt Frankfurt“ und bot „diese Bewohnern der Stadt aber auch Menschen von Außerhalb für eigene Projekte“ (Xqm-Projektgruppe 2011) an. Erst in diesem Prozess wurde der Projektgruppe gewahr, dass das Projekt eine Vielzahl von Menschen störte, die in ihrem Alltag auf die Nutzung des öffentlichen Raumes angewiesen sind. Zu nennen sind auch die Aktivist*innen des „Detroit Black Food Security Network“. Sie verwenden den Begriff Kolumbus-Syndrom nicht für Übergriffigkeiten von Seiten der formalisierten Stadtplanung, welche im hochverschuldeten Detroit auf das Notwendigste zusammengestrichen wurde, sondern für Bevormundungen durch anderen Gruppen, die sich in Detroit im Bereich des urban farming engagieren. Diese setzen sich häufig aus einer jungen, vorwiegend weißen und akademischen Mittelschicht zusammen, die zwar ebenfalls mit geringen finanziellen Mitteln, aber mit einem hohen Vernetzungsgrad und einem großen Know-how in Medienarbeit und Mittelakquise, die Professionalisierung und auch Vermarktlichung von urban farming vorantreiben. Als übergriffig beschreiben die Aktivist*innen den Versuch, Mitglieder des Detroit Black Food Security Network immer wieder in Fragen der Professionalisierung urbaner Landwirtschaft beraten zu wollen, obwohl das urban farming genau von der schwarzen Bevölkerung erfunden wurde, die, aus den Südstaaten für die Industriearbeit nach Michigan kommend, ihre agrarischen Traditionen in der Stadt fortgeführt und in communities bis heute gepflegt haben.

\section{Neue Ansätze performativer Planung}

Vor dem Hintergrund des ausgeprägten Kolumbus-Syndroms der Planung ist es zentral, Partizipation von Betroffenen in Planungsprozessen nicht aufzugeben. Zwar ist es wichtig, sie in ihrer gegenwärtigen Form als Methode zur Schaffung von Zustimmung (vgl. S. 111) zum von den Planer*innen identifizierten,Wesentlichen' (S. 107) sowie als Herrschaftsinstrument (Wagner 2013) zu kritisieren. Genauso fatal aber wäre es, bei dieser Kritik stehen zu bleiben. Das hieße Partizipation als Mittel zur Durchsetzung bürgerlicher Interessen (S. 112) beziehungsweise zur Organisation von Zustimmung zu unternehmerischer Stadtpolitik anzuerkennen und damit als emanzipatorisches Projekt aufzugeben. Wie also muss sich die Partizipation von Betroffenen verändern, um diesem Dilemma zu entgehen? Wie müssen Planungsprozesse aussehen, bei denen Betroffene das ,Wesentliche“ selbst politisch mitbestimmen können?

In jungerer Zeit sind eine Vielzahl neuer ästhetischer und performativer Ansätze entstanden, die nicht dem Büro der Planer*innen entspringen, sondern direkt an den lokalen Verhältnissen ansetzen (Altrock 2014, Kremer 2011). Performative Planung zeichnet sich nach Mackrodt und 
Helbrecht durch einen aktionsorientierten Ansatz aus, bei dem ein Ort durch materielle Praktiken gestaltet wird. Die Planung findet dabei direkt auf der zu verändernden Fläche statt, wobei „das gestalterische Tun am Planungsort im Mittelpunkt der Partizipation“ (Mackrodt/Helbrecht 2013: 15) steht.

„Unter performativer Bürgerbeteiligung verstehen wir Beteiligungsansätze, die über einen längeren Zeitraum (z. B. ein Jahr) im öffentlichen Raum verortet sind und durch ihre physisch-materielle Präsenz ein handlungsorientiertes Beteiligungsangebot im Rahmen eines Stadtentwicklungsprozesses schaffen. BürgerInnen werden aufgefordert - in verschiedenen Projekten auf unterschiedliche Weise - selbst raumgestaltend aktiv zu werden auf einer zu beplanenden Fläche. [...] Die unmittelbare Präsenz der Beteiligungsangebote im öffentlichen Raum trägt zur Gestaltung des zu beplanenden Ortes bei. Dadurch wird für den weiteren Planungsprozess nicht nur eine Debatte über einen Ort geführt, sondern eine gegenwärtige Intervention an einem Ort ermöglicht, die diesen in einem Prozess der Raumgestaltung selbst verändert.“ (Mackrodt/Helbrecht 2013: 16)

Als Beispiele für diese neue Form der Planung nennen Mackrodt und Helbrecht Gemeinschaftsgärten oder die Pioniernutzungen auf dem Tempelhofer Feld in Berlin. Stärker eigeninitiativ und selbstorganisiert interveniert der Do-it-Yourself-Urbanismus direkt und performativ in die Verhältnisse der Stadt Durch durch Guerilla-Gardening, Besetzungen, die Gründung von Kooperativen und Wohnprojekten oder anderen Formen der Raumaneignung schafft er alternative Realitäten in der Stadt, um deren Verstetigung dann in politischen Prozessen gestritten wird (Douglas 2014, Iveson 2013, Bruns 2014, Ring/ Eidner 2013).

Andere Ansätze verfolgen ein Konzept der Zwischennutzung (Buttenberg/ Overmeyer/Spars 2014, Lauinger 2007) oder des Recyclings von Orten im städtischen Wandel (Ziehl 2012). Sie setzen an der Beobachtung an, dass der steigende Verwertungsdruck zu immer kürzeren Verwertungszyklen von Immobilien führt. Bürohochhäuser genügen häufig bereits nach dreißig Jahren nicht mehr den Ansprüchen und dem neuesten Stand der Technik. So werden Leerstand und temporäre urbane Brachen zu einem dauerhaften Bestandteil der sich permanent im Zustand der kreativen Zerstörung befindlichen Städte (Oswalt/Overmeyer/Misselwitz 2013).

Initiativen wie Parc Fiction oder die Planbude in Hamburg organisieren selbst einen öffentlichen Planungsprozess im Sinne einer „kollektiven Wunschproduktion“ (Schäfer/Skene/Hafenrandverein 2008). Urban Catalyst versuchen Nutzungen an Orten aufzuspüren und fortzuschreiben (Oswalt/Overmeyer/Misselwitz 2013). Dem Wem-gehört-die-Stadt-Bündnis in Frankfurt gelang es, den postpolitischen Konsens von Partizipation zumindest selbst zum Thema stadtpolitischer Auseinandersetzungen zu machen (Dzudzek 2013).

Die genannten Initiativen haben spannende neue Ansätze entwickelt, um die Stadt gemeinsam mit denen, die sie alltäglich hervorbringen, neu zu gestalten. Während die einen stark in die Prozesse formal organisierter Stadtplanung eingebunden sind, arbeiten andere autonom. Während die einen neue Ideen entwerfen, setzen andere stärker an den Gegebenheiten und Nutzungen konkreter Orte an und entwickeln diese weiter. 
Viele dieser Initiativen werden stark und zum Teil fast ausschließlich von einer jungen, gebildeten, zumeist weißen urbanen Mittelschicht getragen. Die angewendeten Konzepte kommen häufig aus dem Bereich der Kunst oder einem akademisch geprägten aktivistischen Umfeld (Rauterberg 2013, Ring/Eidner 2013). Nicht selten dienen solche Projekte als Sprungbrett in eine eigene Karriere als kreative Entrepreneure (Buttenberg/Overmeyer/ Spars 2014) oder zur Entwicklung eigenen Wohnraums. So schön eine solche Raumaneignung ist, so muss man doch fragen, wie inklusiv diese zweifelsohne innovativen Formen der performativen Raumproduktionen sind. Es ist daher eine Zukunftsaufgabe, performative Ansätze noch konsequenter auf ihre Machteffekte hin zu befragen. Ansätze des postkolonialen Urbanismus können diese Debatte bereichern.

\section{Postkolonialer Urbanismus}

Dass die Stadt kein unbeschriebenes Blatt ist, auf dem sich unbekümmert neue Entwürfe erproben lassen, sondern ein Ort, der immer schon mit Gegebenheiten und gelebten Praktiken gefüllt ist, bevor er verplant ist, daran erinnern auch neuere Ansätze des postkolonialen Urbanismus. Anaya Roy beispielsweise schreibt den urbanen Subalternen eine eigene political agency zu (Roy 2011a: 235). Sie schaut genau hin, welche Aktivitäten in „peripheries, urban informality, zones of exception, and gray spaces“ (Roy 2011a: 235) stattfinden und welche Impulse diese "spaces at the edge of urban theory“ (Roy 2011a: 235) für die Entwicklung eines anderen Nachdenkens über Stadtentwicklung geben.

„Reclaiming the commons [...] requires not only claiming space but also claiming time, a laying claim to the future. The latter, this orientation to the future, is how we often envision planning. [...] A theory of planning is ultimately about such vectors of space and time. [...] planning itself must be understood as both an everyday and interplanetary practice - of discipline and profession - that is constituted through struggles over space and time.” (Roy 2011b: 14)

Diese „New Geographies of Theory“ (Roy 2009) eines postkolonialen Urbanismus können zentrale Impulse zur Überwindung des Kolumbus-Syndroms von Planung wie auch zur Weiterentwicklung performativer Ansätze liefern. Anstelle Stadt als unbeschriebenes Blatt zu konzeptualisieren, auf dem Architekt*innen, Ingenieur*innen und Planer*innen - aber auch Stadtaktivist*innen - ihre Visionen und Intuitionen ausbreiten können, setzt eine solche Planung an den vorhandenen Praktiken und Nutzungen des Raumes an. Hier kann die Planung des Globalen Nordens von den Praktiken des Globalen Südens lernen: „,G]iving substantially greater attention to documenting urban change on hitherto under-researched cities [enables] learning from practice how to transform the theoretical canon to ensure 21st-century relevance" (Parnell/Robinson 2013: 593). Global betrachtet sind die wenigsten Städte Resultate sauber implementierter Planungspraxis. Im Gegenteil ist die alltägliche Praxis der Planung in den allermeisten Teilen der Welt eine Politik der Regierten (Chatterjee 2007: 53ff.). Sie zeigt sich in der informellen Organisation von Dienstleistungen und der Bereitstellung 
urbaner Infrastruktur ebenso wie in der alltäglichen Aneignung und Nutzung des urbanen Raumes.

In einem solchen Verständnis geht es nicht um den neuen großen Entwurf, sondern um die nachhaltige Weiterentwicklung vorhandener gelebter Kreativität. Diese erfordert kein Papier, sondern das Erkunden des Vorhandenen, die Begegnung und den Dialog im Raum zur Entwicklung des Möglichen. Auf diese Weise werden Betroffene nicht länger zu ,Beplanten“, das heißt zu passiven Objekten von Planungsprozessen degradiert. Stattdessen wäre es die Aufgabe von städtischer wie aktivistischer Planung, ihr professionelles Wissen in den Dienst der Betroffenen zu stellen und sie dabei zu unterstützen ihr Recht auf Stadt zu verwirklichen. Planung sollte Hilfestellung leisten, aus der Informalität oder Illegalität herauszutreten und bestehende Netzwerke, Strukturen, Provisorien und Begegnungsorte zu stabilisieren und verstetigen.

Eine solche Perspektive stärkt die Betroffenen allgemein. Sie stärkt aber besonders die Perspektive derjenigen, die über weniger materielle, diskursive und mediale Ressourcen in Auseinandersetzungen verfügen als die junge, weiße, gebildete Mittelschicht, die das Planungsgeschehen von unten derzeit dominiert.

\section{Wem gehört die Planung? - Stadt erkunden, Planung aneignen}

In Zeiten eines steigenden ökonomischen Verwertungsdrucks in den Städten und der zunehmenden Angleichung von Städten durch die Implementierung global zirkulierender policies wird es zu einer immer wichtigeren Aufgabe städtischer Politik und planerischen Handelns, Freiräume in der Stadt zu erhalten und weiterzuentwickeln. Hierzu müssen partizipative Prozesse, die als Erfüllungsgehilfen eines postpolitischen Konsenses in der unternehmerischen Stadt in Misskredit geraten sind, wieder repolitisiert werden. Daher sollte Burckhardts Frage „Wer plant die Planung?“ die Frage „Wem gehört die Planung?“ zur Seite gestellt werden. Hierzu ist mehr notwendig als das Planen am Schreibtisch oder das Erkunden eines Areals mittels der „Spaziergangswissenschaft“, die Lucius Burckhardt entwickelt hat (Burckhardt 2006). Ob bei der Legalisierung öffentlicher Veranstaltungsräume, der Bewältigung von Brandschutzproblemen oder der Sicherung provisorischer Unterkünfte - Menschen brauchen Hilfestellung, um selbst zu Planer*innen der Stadt zu werden. Stadtplanung sollte ihre Expertise in den Dienst derjenigen stellen, die Stadt bereits gestalten. Dabei sollte sie weder Angst vor Armut, noch vor den aus ihrer Sicht heruntergekommen Ecken der Stadt haben. Stattdessen sollte sie sich Zeit nehmen, die an konkreten Orten stattfinden Praktiken und Raumproduktionen zu verstehen, und fragen, wie sie im Sinne gelebter Urbanität weiterentwickelt werden können. Hierzu können wir von urbanen Praktiken des Globalen Südens ebenso lernen wie von der Wissenschaft des postkolonialen Urbanismus. Die Rolle der Planung bestünde dann auch darin, den Zugang aller Bevölkerungsschichten zum öffentlichen Raum zu gewährleisten. Auf diese Weise ließe sich eine Repolitisierung von Partizipation ebenso verwirklichen wie eine machtsensible Planung im Sinne eines Rechts auf Stadt für alle. 


\section{Autor_innen}

Iris Dzudzek ist Humangeographin. Sie arbeitet zu poststrukturalistischen Theorien, der Regierung der Kreativen Stadt sowie Neuen Geographien der Gesundheit. dzudzek@geo.uni-frankfurt.de

\section{Literatur}

Altrock, Uwe (2014): Das Ende der Angebotsplanung? Instrumente der Planung im Wandel. In: Friederike Maus / Peter Müller / Patrick Küpper / Meike Levin-Keitel / Sara Reimann / Martin Sondermann / Katja Stock / Timm Wiegand (Hg.), Raumentwicklung 3.0 Gemeinsam die Zukunft der räumlichen Planung gestalten. Akademie für Raumforschung und Landesplanung, 15-32.

Bruns, Laura (2014): Stadt selber machen. Ein Handbuch. Berlin: Jovis.

Burckhardt, Lucius (2006): Warum ist Landschaft schön? Die Spaziergangswissenschaft. Kassel: Schmitz.

Burckhardt, Lucius (2013): Der kleinstmögliche Eingriff. Oder die Rückführung der Planung auf das Planbare. Berlin: Schmitz.

Burckhardt, Lucius (2017 [1974]): Wer plant die Planung?. In: s u b \ u r b a n . zeitschrift für kritische stadtforschung, 5, 1-2, 105-114.

Buttenberg, Lisa / Overmeyer, Klaus / Spars, Guido (Hg.) (2014): Raumunternehmen. Wie Nutzer selbst Räume entwickeln. Fallstudien aus der Praxis; Raumstrategien, Entwicklungspfade, Wertschöpfungskonzepte; Diskurse über nutzergetragene Stadtentwicklung. Berlin: Jovis.

Chatterjee, Partha (2007): The Politics of the Governed. Reflections on Popular Politics in Most of the World. New York: Columbia University Press.

Coscarelli, Joe (2014): Spike Lee's amazing rant against gentrification: ,We been here!'. In: Daily Intelligencer, 25.2.2014.

Douglas, Gordon C. C. (2014): Do-it-yourself urban design. The social practice of informal "improvement" through unauthorized alteration. In: City \& Community 13/1, 5-25.

Dzudzek, Iris (2013): Unternehmen oder Unvernehmen? - Über die Krise des Kreativsubjekts und darüber hinaus. In: Geographica Helvetica 68/3, 181-189.

Dzudzek, Iris (2016): Kreativpolitik. Über die Machteffekte einer neuen Regierungsform des Städtischen. Bielefeld: transcript.

Florida, Richard L. (2002): The Rise of the Creative Class. And How It's Transforming Work, Leisure, Community and Everyday Life. New York: Basic Books.

Gomes de Matos, Catarina (2013): Das Modell Barcelona - Partizipation, Protest und Postpolitik. In: s u b $\backslash \mathrm{u}$ r b a n 1/2, 121-140.

Gribat, Nina (2017): Lucius Burckhardt. Der kleinstmögliche Eingriff. In: Frank Eckardt (Hg.), Schlüsselwerke der Stadtforschung. Wiesbaden: Springer, 145-160.

Harvey, David (2009 [1973]): Social Justice and the City. Athen u. a.: University of Georgia Press.

Iveson, Kurt (2013): Cities within the city. Do-it-yourself urbanism and the right to the city. In: International Journal of Urban and Regional Research 37/3, 941-956.

Kremer, Elisabeth (2011): Performative planning as a method of initiating change. In: Sustainable Spatial Development 2. Riga: Riga Technical University, 81-84.

Lauinger, Holger (2007): Urban Pioneers. Berlin : Stadtentwicklung durchZwischennutzung. Berlin: Jovis.

Mackrodt, Ulrike / Helbrecht, Ilse (2013): Performative Bürgerbeteiligung als neue Form kooperativer Freiraumplanung. In: DISP - The Planning Review 49/4, 14-24.

Oswalt, Philipp / Overmeyer, Klaus / Misselwitz, Philipp (2013): Urban Catalyst. The Power of Temporary Use. Berlin: DOM.

Parnell, Susan / Robinson, Jennifer (2013): (Re)theorizing cities from the global south. Looking beyond neoliberalism. In: Urban Geography 33/4, 593-617.

Peck, Jamie (2011): Geographies of policy: From transfer-diffusion to mobility-mutation. In: Progress in Human Geography 35/6, 773-797. 
Prince, Russell (2014): Consultants and the global assemblage of culture and creativity. In: Transactions of the Institute of British Geographers 39/1, 90-101.

Rauterberg, Hanno (2013): Wir sind die Stadt! Urbanes Leben in der Digitalmoderne. Berlin: Suhrkamp.

Ring, Kristien / Eidner, Franziska (2013): Self-Made City. Berlin. Self_Initiated Urban Living and Architectural Interventions. Berlin: Jovis.

Rosol, Marit / Dzudzek, Iris (2014): Partizipative Planung. In: Bernd Belina / Matthias Naumann / Anke Strüver (Hg.), Handbuch kritische Stadtgeographie. Münster: Westfälisches Dampfboot, 212-217.

Roy, Ananya (2009): The 21st-century metropolis. New geographies of theory. In: Regional Studies 43/6, 819-830.

Roy, Ananya (2011a): Slumdog cities: Rethinking subaltern urbanism. In: International Journal of Urban and Regional Research 35/2, 223-238.

Roy, Ananya (2011b): Urbanisms, worlding practices and the theory of planning. In: Planning Theory 10/1, 6--5.

Schäfer, Christoph / Skene, Cathy / Hafenrandverein (2008): Rebellion on level p. In: Charles Esche / Will Bradley (Hg.), Art and Social Change. A Critical Reader. Millbank/ London: Tate Publishing, 283-289.

Schipper, Sebastian (2013): Genealogie und Gegenwart der „unternehmerischen Stadt“. Neoliberales Regieren in Frankfurt am Main 1960 - 2010. Münster: Westfälisches Dampfboot.

Sieverts, Thomas / Ganser, Karl (1993): Vom Aufbaustab Speer bis zur Internationalen Bauausstellung Emscher Park und darüber hinaus. In: DISP - The Planning Review 29/115, 31-37.

Wagner, Thomas (2013): Die Mitmachfalle. Bürgerbeteiligung als Herrschaftsinstrument. Köln: PapyRossa.

Xqm-Projektgruppe (2011): xqm. http://www.jakobsturm.com/xqm (letzter Zugriff am 10.5.2017).

Ziehl, Michael (2012): Second hand spaces. Über das Recyceln von Orten im städtischen Wandel; recycling sites undergoing urban transformation. Berlin: Jovis. 
